\title{
Identification of Extra Chromosomes of Aneuhaploids and Tetrasomics in Rice and the Use of these Aneuploids in Genome Analysis
}

\author{
Z. X. Wang ${ }^{1,2)}$, Osamu Ideta $^{1)}$, Atsushi Yoshimura ${ }^{1)}$, and Nobuo Iwata ${ }^{1, *)}$ \\ ${ }^{1)}$ Plant Breeding Laboratory, Faculty of Agriculture, Kyushu University, Fukuoka 812, Japan \\ ${ }^{2)}$ Present address: Rice Genome Research Program, STAFF Institute, 446-1 Ippaizuka, Kamiyokoba, Tsukuba, Ibaraki 305, Japan \\ * Corresponding author: Dr. Nobuo Iwata, Plant Breeding Laboratory, Faculty of Agriculture, Kyushu University, Fukuoka 812, Japan
}

\begin{abstract}
Summary
Four aneuhaploids $(2 n=13)$ and 5 tetrasomics $(2 n=26)$ of rice (Oryza sativa L.), which were obtained through anther culture of trisomics $(2 n=25)$, were examined by Southern hybridization analysis to identify their extra chromosomes, to test their genetic stability, and to demonstrate their utility in genome alaysis. Twenty-one RFLP markers located on 9 chromosomes were used as probes. Extra chromosome identification was conducted by observing dosage changes of the probes in the aneuhaploids and tetrasomics. The results indicated that the extra chromosomes of the aneuhaploids and tetrasomics were identical to their parental trisomics. The aneuhaploids and tetrasomics used in the study are genetically stable, except the aneuhaploid plant derived from the trisomic with an extra chromosome 5. This plant showed clear DNA variation. Chromosome assignment of unmapped DNA clones was tried by using the identified aneuploids. The result showed that aneuhaploids and tetrasomics are useful materials for genome analysis in rice to assign cloned DNA fragments to specific chromosomes.
\end{abstract}

Key Words: Oryza sativa L., aneuhaploids, tetrasomics, extra chromosomes, RFLP markers, dosage effects.

\section{Introduction}

Several aneuhaploids and tetrasomics of rice (Oryza sati$v a$ L.) have been obtained by means of anther culture of trisomics (Wang and Iwata 1991). They have been classified into groups morphologically, according to the parental trisomics. However, the extra chromosomes of these aneuploids remain to be identified for most of them.

For identifying chromosomes, karyotype analysis of somatic chromosomes and pachytene analysis are usually used. In rice, methods for karyotype analysis of somatic chromosomes (Kurata and Omura 1978, Fukui and Iijima 1991) and pachytene analysis (Kurata et al. 1981, Khush et al . 1984) have been described. However, due to the small size and poor stainablity of the chromosomes, it is difficult to make a complete karyotype analysis in rice. It becomes more difficult for aneuhaploids and tetraso-

Received February 16, $1995 . \quad$ Accepted May 26, 1995.

mics to make a complete karyotype analysis, because they are usually too poor to get enough roots or pollen mother cells for chromosome preparation. Recently, the accomplishment of RFLP maps offers a new tool for breeding and genetic studies (Beckmann and Soller 1986, Dallars 1988, Tanksley et al . 1989). In rice, linkage maps of RFLP markers have been constructed (McCouch et al. 1988, Saito et al, 1991, Kurata et al. 1994). Therefore, molecular markers for each chromosome are available.

In this study, we tried to identify the extra chromosomes of the aneuhaploids and tetrasomics reported in a previous paper (Wang and Iwata 1991) by using RFLP markers, to test their genetical stability, and at the same time to demonstrate their utility in genome analysis of rice.

\section{Materials and Methods}

\section{Aneuhaploid and tetrasomic plants}

Forty-three aneuhaploids and 51 tetrasomics have been obtained from anther culture of 11 types of trisomics in the japonica rice variety "Nipponbare" (Wang and Iwata 1991). The aneuhaploids, as well as the tetrasomics in the progeny of a trisomic are identical to each other morphologically. Aneuhaploids were distinguished into 9 groups, and tetrasomics also into 9 groups morphologically, according to their parental trisomics. Four aneuhaploids and 5 tetrasomics were chosen randomly for this study, and diploid Nipponbare, the original variety, was used as control (Table 1).

\section{DNA probes}

For identifying extra chromosomes of aneuhaploids and tetrasomics, 21 RFLP markers which were located unequivocally on 9 chromosomes were used (Table 2). RFLP markers were the clones from a Pst 1 genomic library of Nipponbare, and all of them are single copy (Saito et al. 1991). Their sizes are from 580 bp to 1,900 bp. For each chromosome 2 or 3 probes were chosen (Table 2).

\section{DNA extraction and hybridization}

DNA was extracted from leaves by a procedure as described in Rogers and Bendich (1988). DNA samples from aneuhaploids, tetrasomics and Nipponbare were digested to completion with one of five restriction en- 
zymes: Bgl II, Dra I, Eco R I, Eco R V, and Hin d III. Southern blotting hybridization were performed according to Southern (1975). Probes were labeled with ${ }^{32} \mathrm{P}$ dCTP by random priming system (Feinberg and Vogel-

Table 1. Aneuhaploids $(2 n=13)$, tetrasomics $(2 n=26)$ and diploid Nipponbare $(2 n=24)$ used in the study

\begin{tabular}{lll}
\hline \hline Line & 2n & Parental trisomics $^{1)}$ \\
\hline AC1566 & 26 & Triplo 1 \\
AC2288 & 13 & Triplo 4 \\
AC2171 & 13 & Triplo 5 \\
AC2472 & 26 & Triplo 6 \\
AC2541 & 26 & Triplo 7 \\
Aha14-1 & 13 & Triplo 9 \\
AC745 & 26 & Triplo 10 \\
AC3182 & 13 & Triplo 11 \\
AC296 & 26 & Triplo 12 \\
Nipponbare & 24 & \\
\hline
\end{tabular}

1): Iwata and Omura, 1984.

Table 2. RFLP markers used in the study and their chromosome locations

\begin{tabular}{cc}
\hline Chromosome & RFLP clones (Npb no.) ${ }^{1)}$ \\
\hline 1 & $54,96,147$ \\
4 & $114, \quad 120,203$ \\
5 & $25,208,251$ \\
6 & 135,200 \\
7 & 91,178 \\
9 & 13,47 \\
10 & 37,60 \\
11 & 44,78 \\
12 & 88,336 \\
\hline
\end{tabular}

1) : Nipponbare clones from Saito et al. (1991).

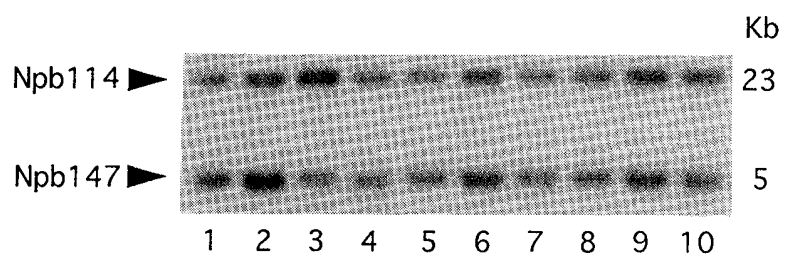

a

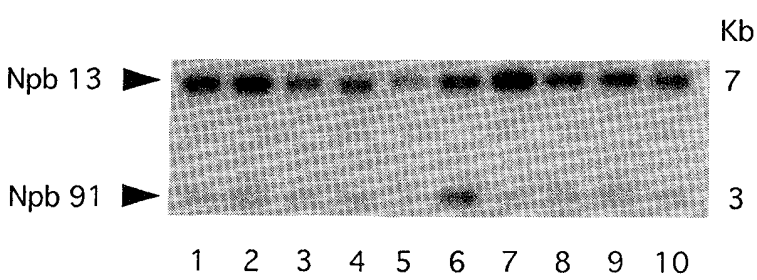

C

Fig. 1. Autoradiogram showing dosage effects in aneuhaploids and tetrasomics, as indicated by the increased signals. Lanes 1 to 10 indicate DNA from Nipponbare, AC1566, AC2288, AC2171, AC2472, AC2541, Aha14-1, AC745, AC3182, and AC296, respectively. a. Npb147 (chromosome 1) showed dosage effect in AC1566 (lanes 2) and Npb114 (chromosome 4) in AC2288 (lane 3); DNA was digested with Dra I. b, Npb208 (chromosome 5) showed dosage effect in AC2171 (lanes 4) and Npb135 (chromosome 6) in AC2472 (lane 5); DNA was digested with Bgl II. c, Npb91 (chromosome 7) showed dosage effect in AC2541 (lane 6) and Npb13 (chromosome 9) in Aha14-1 (lane 7); DNA was digested with Eco R V. d, Npb44 (chromosome 11) showed dosage effect in AC3182 (lanes 9) and Npb336 (chromosome 12) in AC296 (lane 10); DNA was digested with Hin d III. stein 1983). After overnight hybridization at $42^{\circ} \mathrm{C}$, the filters were washed in $6 \times \mathrm{SSC}, 0.2 \%$ SDS for $30 \mathrm{mi}-$ nutes for 2 times at $42^{\circ} \mathrm{C}$, and sequentially in $2 \times \mathrm{SSC}$, $0.2 \%$ SDS for 60 minutes for 1 time at $42^{\circ} \mathrm{C}$. The washed filters were then exposed to $\mathrm{X}$-ray films for 3 to 4 days at $-80^{\circ} \mathrm{C}$, with intensifying screens.

Dosage effect detection and extra chromosome identification If the extra chromsome of a given aneuhaploid or tetrasomic was identical to the chromosome from which a probe DNA fragment originated, the hybridization signal of the probe would become increased in the DNA lane of the aneuhaploid or tetrasomic, compared with other lanes of aneuhaploids, tetrasomics and diploid Nipponbare, because of the dosage effects of its extra chromosomes. Therefore, it is possible to identify extra chromsomes of aneuhaploids and tetrasomics by observing the increased hybridization singal of a known probe in one of the aneuhaploids or tetrasomics.

In such an analysis, the most likely source of error is variation in the quantity of DNAs from aneuhaploids and tetrasomics loaded into each lane of the gel used to prepare the Southern blot. To control this problem, a second clone from different chromosome was used during hybridization to give "within-lane" comparisons, thereby facilitating the identification of an increased hybridization signal in a given aneuhaploid or tetrasomic (Fig. 1, Fig. 2), i.e., two probes located on different chromosomes were mixed and labeled to hybridize to DNAs from aneuhaploids, tetrasomics and Nipponbare. Hybridization signals obtained on autoradiograms were observed visually. The dosage effects were determined by comparing the signals of the probes, within and between the DNA lanes of aneuhaploids, tetrasomics, and diploid Nipponbare.

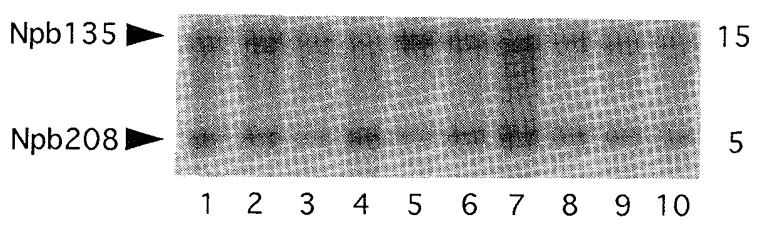

b

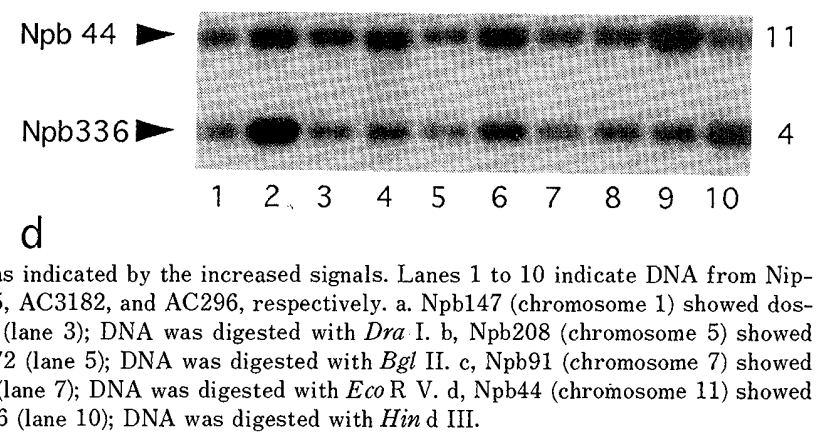


$\mathrm{Kb}$

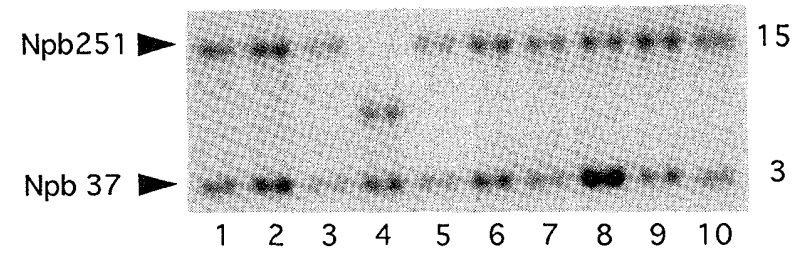

Fig. 2. Autoradiogram of clones of Npb251 (chromosome 5) and Npb37 (chromosome 10) probed onto Eco R I-digested DNAs from Nipponbare, AC1566, AC2288, AC2171, AC2472, AC2541, Aha141, AC745, AC3182, and AC296 (from left to right). Npb37 showed dosage effect in AC745 (lanes 8), and Npb251 gives a hybridization signal shift to low-molecular side (lane 4).

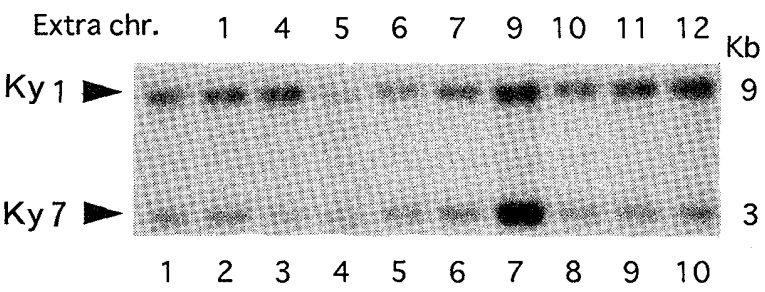

Fig. 3. Autodiogram of clones of $\mathrm{Ky} 1$ and $\mathrm{Ky} 7$ probed onto $\mathrm{Bgl}$ IIdigested DNAs from Nipponbare, AC1566, AC2288, AC2171, AC2472, AC2541, Aha14-1, AC745, AC3182 and AC296 (from left to right). Ky1 was located onto chromosome 4 (lane 3) and Ky7 onto chromosome 9 (lane 7), as indicated by the increased signals.

\section{Assignment of DNA clones}

To demonstrate the utility of rice aneuhaploids and tetrasomics in genome analysis, 10 unmapped DNA clones from a Pst I library of an indica rice variety IR24 (Yoshimura et al., Plant Breeding Laboratory, Kyushu University, unpublished data) were used to assign their chromosomal locations. The methods were the same as described above i.e., two unmapped DNA clones were mixed and labeled to hybridize to DNAs from aneuhaploids and tetrasomics. By observing a change in dosage of a DNA clone in one of aneuhpaloids or tetrasomics, the chromosomal location of the DNA clone was determined.

\section{Results}

\section{Extra chromosomes of aneuhaploids and tetrasomics}

Clone Npb147 (chromosome 1) showed increased hybridization singal in the lane of AC1566 (lane 2 in Fig. 1a), indicating that the extra chromosome of AC1566 was chromosome 1. Likewise, the extra chromosomes of the other aneuhaploids and tetrasomics were found to be identical to those of their parental trisomics (Figs. 1, 2, Table 1).

\section{Genetic stability in aneuhaploids and tetrasomics}

Twenty-one probes and 5 enzymes were successfully used for 27 probe/enzyme combinations in Southern hybridization. The aneuhaploids, as well as the tetrasomics showed the same hybridization patterns as diploid Nipponbare in all of these probe/enzyme combinations, except in the $\mathrm{Npb} 251 / E c o \mathrm{R} \mathrm{V}$ combination, suggesting that there was no DNA variation, such as point mutation, deletion, and insertion in the aneuhaploids and the tetrasomics for the DNA regions having homology with those probes. However, when Npb251 was hybridized to Eco R V-digested DNAs from aneuhaploids and tetrasomics, there was no dosage effect observed, and its hybridization signal shifted to low-molecular side in the DNA lane of AC2171 (lane 4 in Fig. 2), which derived from the trisomic with an extra chromosome 5.

\section{Assignment of DNA clones with aneuhaploids and tetraso-} mics

Fig. 3 showed an autoradiogram of two unmapped clones, Ky1 and Ky7, probed onto Bgl II-digested DNAs from the identified aneuhaploids and tetrasomics. Ky1 was located onto chromosome 4 (lane 3) and Ky7 onto chromosome 9 (lane 7), as indicated by the increased signals. In such a way, we assigned 3 DNA clones (Kyl, $\mathrm{Ky} 4, \mathrm{Ky} 7)$ out of 10 clones examined to specific chromosomes, two to chromosome 4 and one to chromosome 9. Preliminary RFLP mapping data agrees with these assignments (Yoshimura et al., personal communication).

\section{Discussion}

In the present study, we have identified the extra chromosomes of the aneuhaploids and tetrasomics reported in a previous paper (Wang and Iwata 1991), by using RFLP markers. The results showed that the extra chromosomes of each aneuhaploid and tetrasomic are identical to those of their parental trisomics. We have also demonstrated the utility of aneuhaploids and tetrasomics in genome analsyis to assign unmapped DNA clones to specific chromosomes. The result indicated that aneuhaploids and tetrasomics are useful materials for genome analysis in rice.

As somaclonal variation, including point mutation, chromosome number changes and chromosome structure changes, is reported in regenerated plants from tissue culture (Larkin and Scowcroft 1981), it is necessary to make sure whether they were genetically stable, as well as to identify their extra chromosomes when the aneuploids were obtained from tissue culture. In the present study, we used RFLP markers to examine the aneuhaploids and tetrasomics obtained from anther culture of trisomics. In such an analysis, not only the extra chromosomes but also the genetic stability of those aneuploids have been clarified. In the 27 probe/enzyme combinations tested there was no DNA variation, such as point mutation, deletion, and insertion, which should originate from somaclonal variation during the period of anther culture. Our results agree with those of Müller et al. (1990), in which they concluded that there was less DNA variation among the regenerated plants from short incubation periods. The aneuhaploids and tetrasomics used in the present study were also from the calli incubated a short time (about 28 days). This means that 
one could use the regenerated aneuhaploids and tetrasomics from a short incubation period less than one month without testing their genetic stability.

Hybridization signal of Npb251 shifted to lowmolecular side in the DNA lane of AC2171 (lane 4 in Fig. 2), compared with other aneuhaploids, tetrasomics, and diploid Nipponbare, indicating that there should be a DNA variation in AC2171. The DNA variation may originate from the parent trisomic, rather than from the somaclonal variation during the period of anther culture. In fact, AC2171 has one fragmented chromosome in addition to 12 normal chromosomes (Wang and Iwata 1991). We also found that there was some morphological changes in the trisomic from which AC2171 was derived.

Plant aneuploids have been used to assign DNA clones to specific chromosomes and to determine the linkage group-chromosome associations in tomato (Young et al. 1987) and in maize (Helentjaris et al. 1986). In rice, however, only trisomics are available at present. When trisomics were used to assign DNA clones to specific chromosomes by observing change in dosage, it is difficult to determine the dosage effect with certainty, since the difference in 3 versus 2 dosage is not so great. However, as demonstrated in the present study, it becomes easier to determine the dosage effect by using aneuhaploids and tetrasomics, since the dosage difference is 4 versus 2 in tetrasomics and 2 versus 1 in aneuhaploids.

In the plant genome study, it is necessary to detect RFLP by using wide cross combinations, and there is no effective method at present to analyse those DNA clones that show no RFLP with given enzymes in rice. Using rice aneuhaploids or tetrasomics as described in the present study, any single-copy clone could be assigned to its chromosome rapidly and chromosomespecific libraries could be constructed. This should be useful for making up a detailed linkage maps and physical maps of rice chromosomes.

\section{Acknowledgments}

This research was suppported in part by a grant from The Japan Science Society to Z. X. Wang. We thank Dr. A. Saito, Kyushu National Agricultural Experiemnt Station, for his providing us RFLP markers, and Dr. I. Havukkala, STAFF Institute, for his critical reading of the manuscript.

\section{Literature Cited}

Beckmann, J. S. and M. Soller (1986) Restriction fragment length polymorphisms and genetic improvement of agricultural species. Euphytica 35 : 111-124.

Dallas, J. F. (1988) Detection of DNA "fingerprints" of cultivated rice by hybridization with a human minisatellite DNA probe. Proc. Natl. Acad. Sci. USA. 85 : 6831-6835.
Feinberg, A. P. and B. Vogelstein (1983) A technique for radiolabeling DNA restriction endonuclease fragments to high specific activity. Analytical Biochemistry $132: 6-13$.

Fukui, K. and K. Iijima (1991) Somatic chromosome map of rice by imaging methods. Theor. Appl. Genet. $81: 589-596$.

Helentjaris, T., Weber, D. F. and S. Wright (1986) Use of monosomics to map cloned DNA fragments in maize. Proc. Natl. Acad. Sci. USA. 83 : 6035-6039.

Iwata, N. and T. Omura (1984) Studies on the trisomics in rice plants (Oryza sativa L.) VI. An accomplishment of trisomic series in japonica rice plants. Jpn. J. Genet. 59 : 199-204.

Khush, G. S., R. J. Singh, S. C. Sur and A. L. Librojo (1984) Primary trisomics of rice: origin, morphology, cytology and use in linkage mapping. Genetics $107: 141-163$.

Kurata, N. and T. Omura (1978) Karyotype analysis in rice I. A new method for identifying all chromosome pairs. Jpn. J. Genet. $53: 251-255$.

- - - - and N. Iwata (1981) Studies on centromere, chromomere and nucleolus in pachytene nuclei of rice, Oryza sativa, microsporocytes. Cytologia $46: 791-800$.

—-, Y. Nagamura, K. Yamamoto, Y. Harushima, N. Sue, J. Wu, B. A. Antonio, A. Shomura, T. Shimizu, S. Y. Lin, T. Inoue, A. Fukuta, T. Shimano, Y. Kuboki, T. Touyama, Y. Miyamoto, T. Kirihara, K. Hayasaka, A. Miyano, R. Monna, H. S. Žhong, Y. Tamura, Z. X. Wang, T. Momma, Y. Umehara, M. Yano, T. Sasaki and Y. Minobe (1994) A $300 \mathrm{~kb}$ interval rice genetic map including 883 expressed sequences. Nature Genetics $8: 365-372$.

Larkin, P. J. and W. R. Scowcroft (1981) Somaclonal variation-A novel source of variability from cell cultures for plant improvement. Theor. Appl. Genet. $60: 197-214$.

McCouch, S. R., G. Kochert, Z. H. Yu, Z. Y. Wang, G. S. Khush, W. R. Coffman and S. D. Tanksley (1988) Molecular mapping of rice chromosomes. Theor. Appl. Genet. $76: 815-829$.

Müller, E., P. T. H. Brown, S. Hartke and H. Lörz (1990) DNA variation in tissue-culture-derived rice plants. Theor. Appl. Genet. $80: 673-679$.

Rogers, S. O. and A. J. Bendich (1988) Extraction of DNA from plant tissues. Plant Molecular Biology Manual A6 : 1-10.

Saito, A., M. Yano, N. Kishimoto, M. Nakagahra, A. Yoshimura, K. Saito, S. Kuhara, Y. Ukai, M. Kawase, T. Nagamine, S. Yoshimura, O. Ideta, R. Ohsawa, Y. Hayano, N. Iwata and M. Sugiura (1991) Linkage map of restriction fragment length polymorphism loci in rice. Japan. J. Breed. $41: 665-670$.

Southern, N. E. M. (1975) Detection of specific sequences among DNA fragments separated by gel electrophoresis. J. Mol. Biol. 98 : 503-517.

Tanksley, S. D., N. D. Young, A. H. Paternson and M. W. Bonierbale (1989) RFLP mapping in plant breeding: New tools for an old science. Biol / Technology $7: 257-264$.

Wang Z. X. and N. Iwata (1991) Production of N+1 plants and tetrasomics by means of anther culture of trisomic plants in rice (Oryza sativa L.). Theor. Appl. Genet. $83: 12-16$.

Young, N. D., J. C. Miller and S. D. Tanksley (1987) Rapid chromosomal assignment of multiple genomic clones in tomato using primary trisomics. Nuclei Acids Res. 15 : 9339-9348. 